\title{
A note on the sample size determination of sequential and multistage procedures
}

\author{
Kiheon $\mathrm{Choi}^{1}$ \\ ${ }^{1}$ Department of Information and Statistics, Duksung Women's University \\ Received 9 October 2012, revised 25 October 2012, accepted 15 November 2012
}

\begin{abstract}
We particularly emphasized how to determine the number of replications with sequential and multistage procedures. So, the t-test is used to achieve some predetermined level of accuracy efficiently with loss function in the case of normal, chi-squared, and exponential distributions. We provided that the relevance of procedures are sequential procedure, two-stage procedure, modified two-stage procedure, three-stage procedure, and accelerated sequential procedure. Monte Carlo simulation is carried out to obtain the stopping sample size that minimizes the risk.
\end{abstract}

Keywords: Accelerated sequential procedure, modified two-stage procedure, sequential procedure, three-stage procedure, two-stage procedure.

\section{Introduction}

Suppose that we have a sequence of a fixed number of independent observations $X_{1}, \ldots, X_{n}$ from a universe having a $N\left(\mu, \sigma^{2}\right)$ distribution with mean $\mu$ and finite variance $\sigma^{2}$. We wish to test $H_{0}: \mu=\mu_{0}$ against $H_{1}: \mu \neq \mu_{0}$ at the preassigned level $\alpha, 0<\alpha<1$. Then a two-sided test would reject $H_{0}$ if and only if $\left|\sqrt{n}\left(\bar{X}_{n}-\mu_{0}\right) / S_{n}\right|>t_{n-1, \alpha / 2}$ where $\bar{X}_{n}$ is the sample mean, $S_{n}^{2}$ is the sample variance, and $t_{m-1, \alpha / 2}$ is the upper $100(\alpha / 2) \%$ point of the $t$ distribution with $n-1$ degree of freedom. So the size of the test will be exactly $\alpha$. If a population distribution is not normal, then we need the sample size $n$ to be a large sample size. Now, if the test procedure above is used in the case of non-normal distributions with fixed $n$, how different will the true size of the test above be from the nominal level $\alpha$.

We formulate the problem with loss function, and then proposed estimator of the test above in the case of a normal, chi-square, and exponential distributions. In this problem, we argue that sequential, two-stage, modified two-stage, three-stage, and accelerated sequential procedure are provided. These methods are followed by numerical examples and analyses. The procedures presented in this paper is explained with the help of interesting application for design of computer experiments. Statistically designed experiments are considered essential for rapid learning and reducing time to market while preserving high quality and peak performance. Recently, computer simulations have become increasingly popular for running

\footnotetext{
$\dagger$ This work was supported by the Institute of Natural Science Fund, Duksung Women's University, 2012.

1 Professor, Department of Information and Statistics, Duksung Women's University, Seoul 132-714, Korea. E-mail: khchoi@duksung.ac.kr
} 
experiments for the development of products and processes. Issues with computer simulated experiments also common to physical experiments include the need to exploit sequential methods. This new issue has sparked the development of new statistical methods, often known as the design and analysis of computer experiments.

There are two major reasons for using sequential procedures in inference. The first is to decrease expected sample size with regard to hypothesis testing. The second reason is if there is no suitable fixed sample size procedure available. An early estimation example of the latter is Stein's two-stage procedure for estimating the mean of normal distribution with unknown variance, a problem for which no fixed sample size procedure suffices for all possible values of the variance. Usually, purely sequential procedures enjoy many desirable asymptotic properties. However, from a practical point of view, sampling with larger batches can be observed quickly and hence one may be able to cut down the operational time significantly compared with sampling sequentially when time or costs are important design factors. Furthermore, Hall (1981) proposed a triple sampling procedure and showed that the procedure combines the simplicity of Stein (1945) with the efficiency of the fully sequential procedures. In particular, two-stage and three-stage point estimation problems have been studied by Choi (2008, 2009), Ghosh and Mukhopadhyay (1980), Mukhopadhyay (1985), Mukhopadhyay (1985), Hamdy et al. (1988), and Ghosh et al. (1997) in reviewing the progress of multistage estimation methods. Also, Mukhopadhyay et al. (2004) present a wide range of sequential methodology tools and research applications.

The plan of this paper is as follows. Section 2 proposes the sequential procedure, two-stage procedure, modified two-stage procedure, three-stage procedure, and accelerated sequential procedure, and then states the main result of this paper concerning its stopping sample size. Section 3 presents some results of the moderate stopping sample size performance of the procedure using the Monte Carlo method.

\section{Sequential and multistage procedures}

We start with a fixed choice of $n$ and one population distribution function. The true unknown type I error probability is given by

and we define an indicator random variable

$$
p=P\left\{\frac{\sqrt{n}\left(\bar{X}_{n}-\mu_{0}\right)}{S_{n}}>t_{n-1, \alpha / 2}\right\}, 0<p<1,
$$

$$
I=\left\{\begin{array}{l}
1, \text { if } H_{0} \text { is rejected } \\
0, \text { if } H_{0} \text { is not rejected. }
\end{array}\right.
$$

Given a sample of size $n$, one wishes to estimate $p$, by the sample mean $\bar{I}_{n}=n^{-1} \sum_{i=1}^{n} I_{i}$, subject to the loss function

$$
L_{n}=\left(\frac{\bar{I}_{n}}{p}-1\right)^{2}
$$

Note that the squared error loss is not very sensitive where $p$ is small, but the proportional loss is. For fixed $n$ and $p$, the expected loss for (2.1) is given by

$$
E\left[L_{n}\right]=E\left(\frac{\bar{I}_{n}}{p}-1\right)^{2}=\frac{(1-p)}{n p} .
$$


Hence, we may like to estimate $p$ by $\bar{I}_{n}$ in such a way that given a preassigned number $w(>0)$, the risk-bound, our goal is to make the associated risk $E\left[L_{n}\right] \leq w$. It is a very useful goal to achieve, particularly when $w$ is chosen small. The risk is bound from above by $w$, a preassigned positive number, if and only if the sample size $n$ is the smallest integer $\geq w^{-1}(1-p) / p=n^{*}$, say.

In order to estimate $p$, we propose appropriate sequential, two-stage, modified two-stage, three-stage, and accelerated sequential procedure for this problem.

\subsection{Sequential procedure}

Chow and Robbins (1965) proposed a purely sequential procedure for the stopping variable. Let us assume that $p$ is unknown, but $p<p_{0}(<1)$ where $p_{0}$ is known. Then, since $n^{*} \geq w^{-1}\left(1-p_{0}\right) / p_{0}$, we define

$$
m=m(w)=\max \left\{m_{0},\left[w^{-1} \frac{\left(1-p_{0}\right)}{p_{0}}\right]+1\right\}
$$

where $[u]=$ largest integer $<u$ and $m_{0}$ is a fixed integer. Notice that if $w^{-1}\left(1-p_{0}\right) / p_{0}$ is an integer, then $\left[w^{-1}\left(1-p_{0}\right) / p_{0}\right]+1$ will coincide with $w^{-1}\left(1-p_{0}\right) / p_{0}$. Since the magnitude of $n^{*}$ remains unknown, Robbins and Siegmund (1974) considered the following purely sequential stopping variable. We would start with the pilot Bernoulli observations $I_{1}, \ldots, I_{m}$, and then we would continue with one additional observation $I$ at a time and employ the stopping variable

$$
N^{*} \equiv N^{*}(w)=\min \left\{n \geq m: n \geq w^{-} 1 \frac{\left(1-\bar{I}_{n}-n^{-} 1\right)}{\bar{I}_{n}+n^{-1}}\right\}
$$

Notice that $N^{*}(w)$ is well defined and is finite with probability 1. After stopping, the success probability $p$ would be estimated by $\widehat{p}_{N^{*}}=N^{*-1} X_{N^{*}}$ with $X_{N^{*}}=\sum_{i=1}^{N^{*}} I_{i}$ based on the whole observed data $\left(N^{*}, I_{1}, \ldots, I_{N^{*}}\right)$. Obviously, $\widehat{p}_{N^{*}}$ is the sample proportion of $1 \mathrm{~s}$ among $I_{1}, \ldots, I_{N^{*}}$ upon termination.

\subsection{Two-stage procedure}

In some situation, the Robbins-Starr procedure cannot be recommended and a different procedure has to be considered. Now, recall that the sequential procedure (2.2) had started with $m$ initial observations that were followed by drawing one additional observation at a time until termination. Such one-observation-at-a-time sampling may be quite inconvenient in some experimental situations. Sometimes, the data is obtained in batches and it might be more appropriate to use methods which do not require including only one new observation at time. The following is the two-stage sampling procedure in the light of Stein (1945).

Suppose the same setup as before with the optimal fixed-sample size $n^{*}$. Let $m$ be chosen the same as in (2.2) and consider the following two-stage procedure. In the first stage, after $m$ intial or pilot trials, we have observed $\widehat{p}_{m}=m^{-1} X_{m}$, the sample proportion of 1 s, and then define

$$
N_{T S}^{*}(w)=\max \left\{m,\left[w^{-1} \frac{\left(1-\bar{I}_{n}-n^{-1}\right)}{\bar{I}_{n}+n^{-1}}\right]+1\right\} .
$$

If $N_{T S}^{*}(w)=m$, we stop sampling; otherwise, we move to the second stage. In the second stage, if $N_{T S}^{*}(w)>m$, we proceed by recording additional $N_{T S}^{*}(w) \equiv N_{T S}^{*}(w)-m$ observations, all in one single batch. Let $X_{T S}(w)$ be the total number is in the combined sample from both stages. Then $\bar{I}_{N_{T S}^{*}}$ is the final estimator of $p$. 


\subsection{Modified two-stage procedure}

Stein's two-stage procedure tends to overestimate optimal sample size, even asymptotically. This negative feature is due to the initial sample size $m$ which causes big differences in the final sample size $N_{T S}^{*}(w)$. A modified two-stage procedure which improves this feature was suggested by Mukhopadhyay (1980).

The modified two-stage procedure varies the starting-sample size $m$. We choose a real number $\gamma>0$. and define

$$
m=m(w)=\max \left\{2,\left[\left(\frac{1}{w}\right)^{2 /(1+\gamma)}\right]+1\right\} .
$$

We start the experiment with this number of observations and let

$$
N_{M T S}^{*}(w)=\max \left\{m,\left[w^{-1} \frac{\left(1-\bar{I}_{n}-n^{-1}\right)}{\bar{I}_{n}+n^{-1}}\right]+1\right\} .
$$

We implement this Stein's procedure in exactly the same manner as earlier.

\subsection{Three-stage procedure}

The behaviour of Stein's procedure can be improved by adding on additional sampling stage to the algorithm. Hall (1981) introduced the three-stage procedure. The three-stage procedure goes as follows. In the first stage we draw

$$
m=m(w)=\max \left\{2,\left[\left(\frac{1}{w}\right)^{2 /(1+\gamma)}\right]+1\right\}
$$

observations. The number of observations $m$ is controlled by the tuning parameter $\gamma>0$. To determine the intermediate sample size $N_{1}$,

$$
N_{1}^{*}=N_{1}^{*}(w)=\max \left\{m,\left[\rho w^{-} 1 \frac{\left(1-\bar{I}_{m}-m^{-} 1\right)}{\bar{I}_{m}+m^{-1}}\right]+1\right\}
$$

where $0<\rho<1$ is a parameter controlling the sample size in this stage. We sample the difference $N_{1}^{*}-m$ in the second stage if $N_{1}^{*}>m$. Now, based on $I_{1}, \ldots, I_{N_{1}^{*}}$, we define

$$
N_{H}^{*}=N_{H}^{*}(w)=\max \left\{N_{1}^{*},\left[w^{-1} \frac{\left(1-\bar{I}_{N_{1}^{*}}-N_{1}^{*-1}\right)}{\bar{I}_{N_{1}^{*}}+N_{1}^{*-1}}\right]+1\right\} .
$$

In the third stage, the sample size $N_{1}^{*}$ is further augmented, if needed, that is, if $N_{H}^{*}>N_{1}^{*}$, with $N_{H}^{*}-N_{1}^{*}$ additional observations. At the stopped stage, $\theta$ is estimated by $\bar{I}_{N_{H^{*}}}$.

\subsection{Accelerated sequential procedure}

We have seen that the purely sequential procedure can be inconvenient to record observations one-by-one until the process terminates. Thus, it will be useful in practice if we can offer a methodology terminating quickly with its sample size comparing favorably with 
that for a purely sequential strategy. An accelerated sequential procedure first proceeds purely sequentially but continues only part of the way, followed by a batch of the remaining observations gathered in one step.

Hall (1983) first developed an accelerated sequential estimation technique for this problem. Let us choose and fix a number $\rho, 0<\rho<1$. We start with a pilot sample of size $m(\geq 2)$ and followed by one sample at a time as need. The stopping is defined as follows:

$$
\begin{gathered}
t \equiv t(w)=\min \left\{n \geq m ; n \geq \rho w^{-1} \frac{\left(1-\bar{I}_{n}-n^{-1}\right)}{\bar{I}_{n}+n^{-1}}\right\}, \\
N_{1} \equiv N_{1}(w)=\left[w^{-1} \frac{\left(1-\bar{I}_{n}-n^{-1}\right)}{\bar{I}_{n}+n^{-1}}\right]+1, \\
N_{A C} \equiv N_{A C}(w)=\max \left\{t(w), N_{1}(w)\right\} .
\end{gathered}
$$

If $N_{A C}>t$, we sample the difference $N_{A C^{-t}}$ in one single batch. Finally, based on the totality of all samples $I_{1}, \ldots, I_{N_{A C}}$, we estimate $p$ by $\bar{I}_{N_{A C}}$. If $\rho$ is chosen near zero, the accelerated sequential procedure would clearly behave more like the two-stage procedure. But, if $\rho$ is chosen near one, the accelerated sequential procedure would behave like a sequential procedure. In numerous problems, one tends to use $\rho=0.5$.

\section{Monte Carlo studies}

One will note that most theoretical results obtained for the sequential and other multistage procedures are indeed asymptotic in nature. There should be other concerns as well. For example, how does one choose the starting sample size in all these procedures? In this vein, we should add that there is no available optimality criteria in order to pick the best starting sample size. Hence, we tackle this by taking a couple of initial sample size values and examining any visible effects on the moderate sample performances of our procedures. By the same token, the accelerated sequential procedure as well as the three-stage procedure involve the choice of a proper fraction $\rho$, In the accelerated sequential case, we estimate $\rho w^{-1}$ by means of sequential sampling followed by a batch sample, while in the case of the three-stage procedure, we estimate $\rho w^{-1}$ by means of two-stage sampling followed by a batch sample naturally, one would expect that the choice of the $\rho$ value will very likely impact the performances of these procedures for moderate values of $w$. But, again, there is no available optimality criteria yet in order to pick the best $\rho$. Hence, we try various values of $\rho \in(0,1)$ and examine any visible effects or mark on such procedures. Also, how one should choose $\gamma$ of the modified two-stage procedure. In our simulations, we consider $\gamma=0(1) 4$. We accomplish such investigations and comparisons via extensive sets of computer simulations which we summarize here.

We have investigated the performance of the sequential, two-stage, modified two-stage, three-stage, and accelerated sequential procedures on the one sample two-sided testing procedure under a variety of scenarios under the following configuration combinations: $m=5$, $10,20,30,50, m_{0}=100, w=0.1,0.001$ and with the population distribution functions given by Normal with mean 5 and variance 2, exponential with $\lambda=0.01,0.1,1$, and chi-squared distribution with degree of freedom $10,20,30,50$. The entire configurations were repeated 100 times. 
In the following Table 3.1 and 3.2, each represents the average obtained from the 100 replications for a particular value of sample size $m=5,10,20,30,50$ and the chosen value $p_{0}$. The overall features, trends, and conclusions were very similar when we had fixed $w=$ $0.1,0.001$.

From Table 3.1 and 3.2, we make the following assertions: this investigation revealed no detectable common pattern that different choices of $m$, and the choice of $p_{0}$ had no appreciable impact on the final stopping simulation size.

The risk-bound $w$ plays its expected role, that is as the risk decreased, a natural increase in the stopping simulation size could be seen. Under the Normal distribution we found the least variation in the stopping simulation size, followed closely by a similar pattern under chi-squared distribution. The two-stage and three-stage procedures mimics the sequential procedure with respect to the behavior of stopping simulation size.

Table 3.1 Sequential, three-stage and accelerated sequential procedures

\begin{tabular}{|c|c|c|c|c|c|c|c|c|c|}
\hline \multicolumn{10}{|c|}{ Sequential procedure } \\
\hline \multirow{2}{*}{$w$} & \multirow{2}{*}{$p_{0}$} & \multirow{2}{*}{ Normal } & \multicolumn{4}{|c|}{ Chisq } & \multicolumn{3}{|c|}{ Exp } \\
\hline & & & $\mathrm{df}=10$ & $\mathrm{df}=20$ & $\mathrm{df}=30$ & $\mathrm{df}=50$ & $\lambda=0.01$ & $\lambda=0.1$ & $\lambda=1$ \\
\hline \multirow{3}{*}{0.1} & 0.06 & 192.4 & 176.4 & 185.4 & 185.4 & 185.6 & 160.8 & 161 & 160.6 \\
\hline & 0.10 & 180.2 & 161.9 & 167.3 & 173.7 & 174.5 & 120.8 & 119.4 & 119 \\
\hline & 0.30 & 180.6 & 161.3 & 166.3 & 169.5 & 172.7 & 120.7 & 120.9 & 121.6 \\
\hline \multirow{3}{*}{0.001} & 0.06 & 19024.8 & 16663.1 & 17555.7 & 17949 & 18352.4 & 15667 & 15667 & 15667 \\
\hline & 0.10 & 19006.0 & 16363.6 & 17528.9 & 17962 & 18402 & 11278.8 & 11334.2 & 11289.4 \\
\hline & 0.30 & 19005.5 & 16314.8 & 17514.7 & 17948.7 & 18429.1 & 11005.9 & 10998.4 & 10979 \\
\hline \multicolumn{10}{|c|}{ Three-stage procedure $(\rho=0.1)$} \\
\hline \multirow{2}{*}{$w$} & \multirow{2}{*}{$p_{0}$} & \multirow{2}{*}{ Normal } & \multicolumn{4}{|c|}{ Chisq } & \multicolumn{3}{|c|}{ Exp } \\
\hline & & & $\mathrm{df}=10$ & $\mathrm{df}=20$ & $\mathrm{df}=30$ & $\mathrm{df}=50$ & $\lambda=0.01$ & $\lambda=0.1$ & $\lambda=1$ \\
\hline \multirow{3}{*}{0.1} & 0.06 & 198.3 & 185 & 190 & 189.1 & 197.3 & 161.6 & 161.3 & 160.9 \\
\hline & 0.10 & 191.6 & 167.8 & 176 & 187 & 179 & 119.7 & 122.5 & 123.9 \\
\hline & 0.30 & 183.7 & 165.7 & 173.7 & 177.7 & 184.7 & 122.3 & 122.4 & 121.4 \\
\hline \multirow{3}{*}{0.001} & 0.06 & 19003.6 & 16640 & 17569.3 & 17916.1 & 18386.9 & 15667 & 15667 & 15667 \\
\hline & 0.10 & 18994.3 & 16325.6 & 17598.2 & 17943.3 & 18391.4 & 11314 & 11351.6 & 11349.2 \\
\hline & 0.30 & 18930.9 & 16299.1 & 17582.6 & 17927.7 & 18359.3 & 11019.9 & 10982.2 & 11003.6 \\
\hline & & & & ree-stage & cedure ( & $0.4)$ & & & \\
\hline$w$ & $p_{0}$ & Normal & & & & & & Exp & \\
\hline & $p_{0}$ & Normal & $\mathrm{df}=10$ & $\mathrm{df}=20$ & $\mathrm{df}=30$ & $\mathrm{df}=50$ & $\lambda=0.01$ & $\lambda=0.1$ & $\lambda=1$ \\
\hline & 0.06 & 195.8 & 179.6 & 191 & 188.4 & 193.1 & 161.6 & 160.5 & 161.4 \\
\hline 0.1 & 0.10 & 180.2 & 157.1 & 166.6 & 171.9 & 176.3 & 121.3 & 120.8 & 120.6 \\
\hline & 0.30 & 179 & 160.4 & 166.8 & 169.2 & 169 & 121.5 & 121.4 & 118.1 \\
\hline & 0.06 & 18993.2 & 16633.7 & 17603.9 & 18026.9 & 18430.4 & 15667 & 15667 & 15667 \\
\hline 0.001 & 0.10 & 18960.1 & 16372.6 & 17559.3 & 18006.5 & 18418.5 & 11337 & 11299.9 & 11337.7 \\
\hline & 0.30 & 18962 & 16319.9 & 17576.6 & 17927.5 & 18275.7 & 10929.7 & 10997.4 & 10975.3 \\
\hline & & & & ree-stage & cedure ( & $0.7)$ & & & \\
\hline$w$ & & Normal & & & & & & Exp & \\
\hline$w$ & $p_{0}$ & Normal & $\mathrm{df}=10$ & $\mathrm{df}=20$ & $\mathrm{df}=30$ & $\mathrm{df}=50$ & $\lambda=0.01$ & $\lambda=0.1$ & $\lambda=1$ \\
\hline & 0.06 & 195.9 & 179.2 & 184 & 190.4 & 188.7 & 161.8 & 161.9 & 161.1 \\
\hline 0.1 & 0.10 & 180.3 & 156.3 & 163.4 & 165.1 & 173.2 & 119 & 120.3 & 119.6 \\
\hline & 0.30 & 177.1 & 153.8 & 172.3 & 166.9 & 170.2 & 117.7 & 118.7 & 117.3 \\
\hline & 0.06 & 19030 & 16598 & 17556.1 & 17992.1 & 18315.1 & 15667.3 & 15667.1 & 15667.6 \\
\hline 0.001 & 0.10 & 18994.9 & 16226.1 & 17575.4 & 17923.3 & 18417.9 & 11311.7 & 11298.5 & 11333.3 \\
\hline & 0.30 & 18919 & 16308.1 & 17519.3 & 17968 & 18414.2 & 10973.4 & 10980.1 & 10962 \\
\hline & & & & ree-stage & cedure ( & $\overline{0.9)}$ & & & \\
\hline 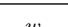 & & & & & & & & Exp & \\
\hline$w$ & $p_{0}$ & Normal & $\mathrm{df}=10$ & $\mathrm{df}=20$ & $\mathrm{df}=30$ & $\mathrm{df}=50$ & $\lambda=0.01$ & $\lambda=0.1$ & $\lambda=1$ \\
\hline & 0.06 & 195.9 & 180.3 & 182.1 & 193.1 & 194.7 & 161.5 & 160.9 & 161.5 \\
\hline 0.1 & 0.10 & 191.9 & 176.3 & 170.1 & 174.6 & 180.9 & 119.6 & 118.7 & 119.9 \\
\hline & 0.30 & 190.8 & 166.8 & 178 & 177 & 202.4 & 120.4 & 124.5 & 123.2 \\
\hline & 0.06 & 18964.2 & 16624.7 & 17526.8 & 17921.3 & 18388.9 & 15667 & 15667.4 & 15667.4 \\
\hline 0.001 & 0.10 & 18967.3 & 16364.8 & 17471.4 & 18014.3 & 18392.5 & 11312.5 & 11301.2 & 11298.6 \\
\hline & 0.30 & 19114 & 16350.2 & 17548.9 & 18066.7 & 18427.8 & 10973.4 & 11009.2 & 11002.1 \\
\hline & & & Acce & ted seque & al proced & $(\rho=0.5)$ & & & \\
\hline 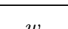 & & & & & & & & Exp & \\
\hline$w$ & $p_{0}$ & Normal & $\mathrm{df}=10$ & $\mathrm{df}=20$ & $\mathrm{df}=30$ & $\mathrm{df}=50$ & $\lambda=0.01$ & $\lambda=0.1$ & $\lambda=1$ \\
\hline & 0.1 & 184.3 & 162.1 & 669.8 & 910.1 & 1013 & 120.5 & 416.3 & 508.2 \\
\hline & 0.4 & 382.2 & 222.6 & 682.3 & 903.2 & 984.6 & 121.9 & 424.8 & 498.6 \\
\hline 0.1 & 0.7 & 693.4 & 368.6 & 696.6 & 934.5 & 995.4 & 195.1 & 491.6 & 515.3 \\
\hline & 0.9 & 788.8 & 550.7 & 811.7 & 942.4 & 1026.8 & 321.2 & 498 & 515.3 \\
\hline & 0.1 & 21499 & 18884.2 & 31006.3 & 36345.7 & 39911 & 12102.5 & 20832.7 & 21856.2 \\
\hline & 0.4 & 20933.6 & 17320.5 & 29426.7 & 34780.1 & 39109.5 & 11307.1 & 19677 & 22014.1 \\
\hline 0.001 & 0.7 & 23932.8 & 19545.1 & 29489.2 & 34922.8 & 39215.6 & 13186.1 & 19812.7 & 21573 \\
\hline & 0.9 & 28774.1 & 24199.4 & 31367.2 & 36286 & 39390.6 & 16745.2 & 20184.1 & 21843 \\
\hline
\end{tabular}


Let us now compare the procedures in Table 3.1 with one another. One can easily see that the sequential procedure looks better than the other two procedures. This should not be very surprising, because for the sequential procedure we update our estimators after each sample, and after each sample we decide whether to take another sample or not. However, this very property of bookkeeping after every sample makes the sequential procedure operationally inconvenient and increases the cost of operation and implementation. So, to cut down the cost of operation and to make the procedure more easily implementable and yet to preserve the second order asymptotic properties, the accelerated sequential procedure and the threestage procedure come into the picture as viable competitors.

Table 3.2 Two-stage and three-stage procedures

\begin{tabular}{|c|c|c|c|c|c|c|c|c|c|}
\hline \multicolumn{10}{|c|}{ Two-stage procedure } \\
\hline \multirow{2}{*}{$w$} & \multirow{2}{*}{$p_{0}$} & \multirow{2}{*}{ Normal } & \multicolumn{4}{|c|}{ Chisq } & \multicolumn{3}{|c|}{ Exp } \\
\hline & & & $\mathrm{df}=10$ & $\mathrm{df}=20$ & $\mathrm{df}=30$ & $\mathrm{df}=50$ & $\lambda=0.01$ & $\lambda=0.1$ & $\lambda=1$ \\
\hline \multirow{3}{*}{0.1} & 0.06 & 204.5 & 180.8 & 194.1 & 194.1 & 191.3 & 163.7 & 161.9 & 161.3 \\
\hline & 0.10 & 188.2 & 167.1 & 172.3 & 177 & 181.3 & 120.3 & 119.2 & 121.9 \\
\hline & 0.30 & 192.4 & 157.3 & 169.8 & 180 & 186.7 & 119.9 & 123.1 & 124.4 \\
\hline \multirow{3}{*}{0.001} & 0.06 & 18947 & 16626.6 & 17532.1 & 18011.5 & 18359.8 & 15667.1 & 15667.1 & 15667 \\
\hline & 0.10 & 18967.3 & 16318.3 & 17483.9 & 17971.4 & 18397.8 & 11324.9 & 11335.0 & 11298.7 \\
\hline & 0.30 & 19046.3 & 16296.1 & 17491.3 & 17927.8 & 18378.3 & 10978.8 & 11008.0 & 11008 \\
\hline \multicolumn{10}{|c|}{ Modified two-stage procedure $(\gamma=0)$} \\
\hline \multirow{2}{*}{$w$} & & Normal & \multicolumn{4}{|c|}{ Chisq } & \multicolumn{3}{|c|}{$\operatorname{Exp}$} \\
\hline & $p_{0}$ & Normal & $\mathrm{df}=10$ & $\mathrm{df}=20$ & $\mathrm{df}=30$ & $\mathrm{df}=50$ & $\lambda=0.01$ & $\lambda=0.1$ & $\lambda=1$ \\
\hline \multirow{3}{*}{0.1} & 0.06 & 67.7 & 66.1 & 66.9 & 67.8 & 66.9 & 56.4 & 55 & 60.2 \\
\hline & 0.10 & 69 & 64.7 & 67.1 & 68.5 & 69.7 & 56.6 & 56.4 & 57.3 \\
\hline & 0.30 & 67.6 & 66.3 & 68.9 & 68.3 & 67.2 & 56.6 & 56.9 & 57.4 \\
\hline \multirow{3}{*}{0.001} & 0.06 & 18875.7 & 16335.5 & 17628 & 18144.8 & 18288 & 11035 & 10902 & 11109.4 \\
\hline & 0.10 & 18839.6 & 16297.8 & 17590.7 & 17898.7 & 18542.2 & 10991.1 & 10956.9 & 10980.5 \\
\hline & 0.30 & 19104 & 16320.5 & 17618.3 & 17974.6 & 18400.2 & 10938.9 & 10998.9 & 11050.6 \\
\hline \multicolumn{10}{|c|}{ Modified two-stage procedure $(\gamma=1)$} \\
\hline \multirow{2}{*}{$w$} & \multirow[b]{2}{*}{$p_{0}$} & Normal & & & & & & $\operatorname{Exp}$ & \\
\hline & & Normal & $\mathrm{df}=10$ & $\mathrm{df}=20$ & $\mathrm{df}=30$ & $\mathrm{df}=50$ & $\lambda=0.01$ & $\lambda=0.1$ & $\lambda=1$ \\
\hline & 0.06 & 19.7 & 19 & 19.8 & 19.4 & 19.4 & 18.2 & 18.1 & 18.1 \\
\hline 0.1 & 0.10 & 19.3 & 19.4 & 19.5 & 19.4 & 19.7 & 17.8 & 17.9 & 17.8 \\
\hline & 0.30 & 19.8 & 19.5 & 19.2 & 19.3 & 19.8 & 18.2 & 18.4 & 18.1 \\
\hline & 0.06 & 15188.9 & 13488.6 & 14667.2 & 14667.9 & 15417.2 & 9995.5 & 9456 & 9705.9 \\
\hline 0.001 & 0.10 & 14811.5 & 14050.1 & 13452 & 14331.6 & 14445.7 & 10114.2 & 9775.6 & 9979.3 \\
\hline & 0.30 & 14642.7 & 13398.4 & 14322.6 & 13947.3 & 15424.2 & 9740.9 & 9909.4 & 10475 \\
\hline & & & & fied two- & ge proced & $(\gamma=2)$ & & & \\
\hline & & & & & & & & Exp & \\
\hline$w$ & $p_{0}$ & Normal & $\mathrm{df}=10$ & $\mathrm{df}=20$ & $\mathrm{df}=30$ & $\mathrm{df}=50$ & $\lambda=0.01$ & $\lambda=0.1$ & $\lambda=1$ \\
\hline & 0.06 & 10.9 & 11 & 10.9 & 11 & 10.7 & 10.3 & 10.3 & 10 \\
\hline 0.1 & 0.10 & 11 & 10.8 & 11.3 & 11.1 & 11 & 10.6 & 10.2 & 10.6 \\
\hline & 0.30 & 11 & 10.9 & 10.6 & 11.1 & 11.2 & 10.1 & 10.1 & 10.2 \\
\hline & 0.06 & 6820.7 & 6340.1 & 6645.7 & 6642.4 & 6748.1 & 5592.4 & 5456.7 & 5909.4 \\
\hline 0.001 & 0.10 & 6702.4 & 6614.1 & 6740.7 & 6843.4 & 6836.7 & 5572.1 & 5581.8 & 5594.4 \\
\hline & 0.30 & 6775.7 & 6489.7 & 6709.3 & 6490.1 & 7002.4 & 5712.1 & 5511.8 & 5508.4 \\
\hline & & & & fied two- & ge proced & $(\gamma=3)$ & & & \\
\hline$w$ & & Normal & & & & & & Exp & \\
\hline & $p_{0}$ & Normal & $\mathrm{df}=10$ & $\mathrm{df}=20$ & $\mathrm{df}=30$ & $\mathrm{df}=50$ & $\lambda=0.01$ & $\lambda=0.1$ & $\lambda=1$ \\
\hline & 0.06 & 9.2 & 9.1 & 9.1 & 9.2 & 9.1 & 8.6 & 8.6 & 8.5 \\
\hline 0.1 & 0.10 & 9.1 & 9.2 & 9.2 & 9 & 9.1 & 9 & 8.7 & 8.6 \\
\hline & 0.30 & 9.2 & 9.1 & 9.2 & 9 & 9.1 & 8.5 & 8.8 & 8.8 \\
\hline & 0.06 & 3809.7 & 3839.1 & 3880.2 & 4018.5 & 3937.5 & 3605 & 3420.5 & 3491.4 \\
\hline 0.001 & 0.10 & 3804.2 & 3828.9 & 3760.2 & 3854.1 & 3945.3 & 3396.2 & 3428.6 & 3440.6 \\
\hline & 0.30 & 4056.1 & 3931 & 3891.2 & 3877.5 & 3944.4 & 3499.2 & 3582.6 & 3634.4 \\
\hline & & & & fied two- & ge proced & $\gamma=4)$ & & & \\
\hline$w$ & & & & & & & & Exp & \\
\hline$w$ & $p_{0}$ & Normal & $\mathrm{df}=10$ & $\mathrm{df}=20$ & $\mathrm{df}=30$ & $\mathrm{df}=50$ & $\lambda=0.01$ & $\lambda=0.1$ & $\lambda=1$ \\
\hline & 0.06 & 9.2 & 9 & 9 & 9.3 & 9.2 & 8.7 & 8.7 & 8.6 \\
\hline 0.1 & 0.10 & 9.2 & 9.2 & 9.2 & 9.2 & 9.2 & 7.6 & 8.8 & 8.7 \\
\hline & 0.30 & 9.2 & 9.3 & 9.2 & 9.1 & 9.3 & 8.7 & 8.4 & 8.7 \\
\hline & 0.06 & 2723.7 & 2657.8 & 2653.3 & 2635.1 & 2703.7 & 2412.4 & 2437.6 & 2382.6 \\
\hline 0.001 & 0.10 & 2610.4 & 2669.4 & 2612.4 & 2656.4 & 2683 & 2446.7 & 2410.1 & 2453.8 \\
\hline & 0.30 & 2701.9 & 2433.9 & 2653.3 & 2633.9 & 2646.7 & 2411.6 & 2436.8 & 2459.6 \\
\hline
\end{tabular}

In Table 3.2, we present a summary of our findings in connection with the two-stage and the modified two-stage procedure. In the case of the modified two-stage procedure, a small 
$\gamma$ will lead to a large value of stopping simulation size. The point is that we wish to give some useful recommendations about $\gamma$ which are not too small. The various entries are self explanatory.

In the case of the two-stage and modified two-stage procedures, the choice of the starting sample size was a crucial factor in achieving good performances of these techniques in non asymptotic situations. This was the case because the common value was to be estimated only once, based on first stage or pilot sample sizes, and there was no possibility of revision or updating such estimators in the follow up. The sequential, accelerated sequential and the three-stage sampling procedures are very different on this count. In the sequential procedure, the estimators are updated with every new observation from each population arriving sequentially. So, as long as the starting sample size is not very large, one will essentially do just fine because even a wrongfully chosen small starting sample size is going to be checked and updated again and again, throughout the sampling process. In other words, it is quite alright to be conservative here and pick a small starting sample size. Similar arguments or their variants are also valid in the contexts of both the accelerated sequential and three-stage procedures. In summary, we add that these three multistage procedures are fairly robust with respect to the starting sample size.

Our remarks from the previous indicate little in the performance of the sequential, the two-stage procedure, the modified two-stage procedure, the three-stage procedure, and accelerated sequential procedure as measured by their stopping simulation sizes. It should be obvious that each procedure has its own set of strengths and weaknesses. Hence, it is not possible to declare a clear winner. On account of such extreme similarities across simulations, our recommendation of one procedure over the other relies heavily on the determination of which procedure runs more efficiently. The modified two-stage procedure terminated with a stopping simulation size that was smaller than that required by the corresponding the other procedures. The suggestions offered here have not taken into account any kind of detailed cost-benefit analysis. Our suggestions are essentially guided by only very rudimentary operations of each procedure.

\section{References}

Choi, K. (2008). Two-stage procedure with lower bound variance based on bootstrap. Journal of the Korean Data \& Information Science Society, 19, 657-666.

Choi, K. (2009). Minimum risk point estimation of two-stage procedure for mean. Journal of the Korean Data $\&$ Information Science Society, 20, 887-894.

Chow, T. S. and Robbins, H. (1965). On the asymptotic theory of fixed width sequential confidence intervals for the mean. The Annals of Mathematical Statistics, 36, 457-462.

Ghosh, M. and Mukhopadhay, N. (1981). Consistency and asymptotic efficiency of two-stage and sequential estimation procedure. Sankya A, 43, 220-227.

Ghosh, M., Mukhopadhay, N. and Sen, P. K. (1997). Sequential estimation, John Wiley, New York.

Hall, P. (1981). Asymptotic theory of triple sampling for estimation of a mean. Annals of Statistics, 9, $1229-1238$.

Hall, P. (1983). Sequential estimation saving sampling operations. Journal of the Royal Statistical Society $B, \mathbf{4 5}, 219-223$.

Hamdy, H. I., Mukhopadhyay, N., Costanza, M. C. and Son, M. S. (1988), Triple stage point estimation for exponential location parameter. Annals of the Institute of Statistical Mathematics, 40, 785-797.

Mukhopadhyay, N. (1980), A consistent and asymptotically efficient two-stage procedure to construct fixedwidth confidence intervals for the mean. Metrika, 27, 281-284. 
Mukhopadhyay, N. (1985), A note on three-stage and sequential point estimation procedures for a normal mean. Sequential Analysis, 4, 311-319.

Mukhopadhyay, N. Datta, S. and Chattopadhyay S. (2004), Applied sequential methodologies, Marcel Dekker, New York.

Stein, C. (1945), A two sample test for a linear hypothesis whose power is independent of the variance. The Annals of Mathematical Statistics, 16, 243-258. 